\title{
Fractional-order two-input two-output process identification based on Haar operational matrix
}

\section{Kajal Kothari \& Utkal Mehta}

To cite this article: Kajal Kothari \& Utkal Mehta (2021) Fractional-order two-input two-output process identification based on Haar operational matrix, International Journal of Systems Science, 52:7, 1373-1385, DOI: 10.1080/00207721.2020.1857503

To link to this article: https://doi.org/10.1080/00207721.2020.1857503

曲 Published online: 21 Dec 2020.

Submit your article to this journal $\widetilde{x}$

Џll Article views: 67

Q View related articles $\sqsubset$

View Crossmark data \lceil 


\title{
Fractional-order two-input two-output process identification based on Haar operational matrix
}

\author{
Kajal Kothari (D) and Utkal Mehta (D) \\ School of Engineering and Physics, The University of the South Pacific (USP), Suva, Fiji Islands
}

\begin{abstract}
The identification of multi-input-multi-output process is always challenging due to significant loop interactions. This article proposes an identification technique for two-input-two-output processes with fractional order models via Haar wavelet feature. The presented technique can estimate independent four fractional single-pole time delay models without additional steps to decouple processes during identification. The method uses a well-known relay feedback test to produce the input-output data for measurements. Then, the Haar wavelet operational matrix based algebraic approach is utilised to identify the unknown process models with reduced complexity. Numerical analysis on various examples show the efficacy even in presence of noise and without additional filter or preprocessing of measured signals. The comparative study proves advantages of the presented approach.
\end{abstract}

\section{ARTICLE HISTORY}

Received 15 January 2019

Accepted 22 November 2020

\section{KEYWORDS}

TITO process; fractional-order models; identification; Haar wavelet; operational matrix

\section{Introduction}

In recent years, fractional calculus (FC) has captured a huge attention in system identification and modelling with fractional power differentiation. This is because of significantly improved accuracy and applications in science and engineering in contrast to conventional (integer) calculus (Monje et al., 2010; Petráš, 2011). The integro-differential equations with an arbitrary or even complex order of operation facilitate additional flexibility and extra degree of freedom for dynamic process modelling even with lowerorder models. Researchers have also proposed several fractional-order (non-integer) controllers, most commonly said a fractional-order-proportional-integralderivative (FOPID). On the same fact a tremendous potentials has been shown in the area of fractionalorder system identification where accuracy and fractional behaviour to be captured by any order transfer function model. In particular, fractional order linear time invariant single-input single-output (SISO) systems have been reported recently and have shown some most attractive results. Literature, Li et al. (2015), Kothari et al. (2018a), Tang et al. (2017), Pandit et al. (2017), Mittal and Pandit (2018), and Kothari et al. (2018b) are some recent publications on applications of operational matrices or Haar wavelets for parameter identification of fractional and/or integer order transfer functions.

Apart from SISO, many sophisticated processes in industry exhibit multivariable nature. These processes are complex owing to multiple inputs multiple outputs and their loop interactions which makes the identification of multi-input multi-output (MIMO) process difficult and time consuming. Due to strong interaction between outputs and inputs, such processes are more complex to study than the single-input singleoutput (SISO). When some processes are not suitable to test in open-loop operation due to instability, safety and reliability issues, a closed-loop identification strategy is more preferable to extract the process dynamics. In this work, we have considered the most general subcategory of MIMO process that is two-input twooutput (TITO) process with particularly emphasising the fractional-order (FO) process identification.

Various identification techniques have been proposed in the literature for MIMO or TITO processes

CONTACT Kajal Kothari s11151029@student.usp.ac.fj E School of Engineering and Physics, The University of the South Pacific (USP), Laucala campus, Suva, Fiji Islands 
based on integer low-order models. Most commonly the processes were modelled as a pair of first-order integer transfer functions for TITO dynamics. Among these strategies, a relay feedback technique and variances in relay with type of tests are shown in studies (Bajarangbali \& Majhi, 2012; Berner, Soltesz, Åström, et al., 2017; Berner, Soltesz, Hägglund, et al., 2017; Kalpana et al., 2015; Nema \& Padhy, 2015; Padhy \& Majhi, 2006; Semino \& Scali, 1998). The improved identification for MIMO processes was described firstly in Semino and Scali (1998). Same way, a TITO process was approximated using a critical gain and frequency of oscillations by Padhy and Majhi (2006) and further revisited in Bajarangbali and Majhi (2012). A non-square MIMO system was identified using time-domain relay response expressions in Kalpana et al. (2015). A state-space based time domain strategy for TITO process was illustrated in Nema and Padhy (2015) to identify TITO FOPDT models. Berner, Soltesz, Hägglund, et al. (2017) described autotuner TITO process identification based on a relay method. Their method uses only single relay test for four individual transfer functions and also does not need to wait for convergence, so makes identification faster. Again authors have evaluated practically a relay autotuner with short excitation on quadruple tank setup (Berner, Soltesz, Åström, et al., 2017). Besides relay test, some alternative techniques have been reported to identify TITO processes. Say for example, applied source separation method (Broman et al., 1999), curve fitting and genetic algorithm based method (Viswanathan et al., 2001), a closed-loop reaction curve method (Ram \& Chidambaram, 2014) and non-linear least square minimisation method (Ram \& Chidambaram, 2016) have been well explained in literature. A commonly adopted technique using step response data was developed for parameter identification with a sequence of setpoint step signals (S.-Y. Li et al., 2005a, 2005b; Pereira et al., 2017). A step response test with particle swarm optimisation and search algorithm for multivariable process was presented by Jin et al. (2012). Real-time multivariable systems were identified in literature say for example; dual-actuator hard disk-drive (Zheng et al., 2005), twin rotor MIMO systems (Chalupa et al., 2015; Tastemirov et al., 2017) and a hydraulic canal (San-Millan et al., 2017). Even though much has been discussed for TITO processes, a very few study has been discussed for fractional-order identification applied for multivariable or TITO processes. Recently, a decoupled method for fractional-order TITO processes in Li and Chen (2014) and a multivariable model based on fractional-order transfer functions with a delay in San-Millan et al. (2017) have shown clearly a superior performance. This study was carried out to demonstrate how non-integer models do help to understand the complex processes accurately.

Owing to complex nature of both, highly coupled TITO process and fractional calculus, how to deal with identification problem in fractional-order with less complexity and better accuracy is an open problem. In this article, TITO process is considered with no prior knowledge of its counterpart integerorder model or structure. The work is more desirable for coupled processes, where it does not take numerous tests and not only depend on a single ultimate frequency point. The presented method uses a well adopted relay feedback test to generate inputoutput responses in a closed-loop and relay with $\mathrm{P}$ controller induce a sustained oscillation to stimulate the TITO process. The technique can estimate the process parameters by performing two closed-loop relay experiments without decentralised process. Using simple measurable responses four independent fractionalorder models for considered TITO process have been obtained. We understand that a fractional-order differential equation can increase computational complexity due to non-integer order, however this method converts the complex integral-differential equations of any orders into simple algebraic equations. Even the proposed technique can consequently facilitates simultaneous estimation of delay, differentiation orders and other model parameters. It can estimate four independent fractional-order models without additional efforts to decouple the process and also without filtering the noisy measured data. The method is therefore faster to apply in TITO processes and can extend easily to multivariable processes. Finally, this new technique is successfully verified on relevant process model examples.

\section{Fractional order TITO process}

Let the input-output relationship of a coupled TITO process is expressed as,

$$
\left[\begin{array}{l}
y_{1} \\
y_{2}
\end{array}\right]=\left[\begin{array}{ll}
g_{11} & g_{12} \\
g_{21} & g_{22}
\end{array}\right]\left[\begin{array}{l}
u_{1} \\
u_{2}
\end{array}\right]
$$


In aforementioned expression, the TITO process can be considered as four individual transfer functions and each independent process $g_{i j}(i, j=1,2)$ can be characterised using continuous-time differential equation with positive real order. It can be written most commonly as

$$
\sum_{p=0}^{n} a_{p} \mathscr{D}^{\alpha_{p}} y(t)=\sum_{q=0}^{m} b_{q} \mathscr{D}^{\beta_{q}} u(t-L)
$$

where $a_{p}(p=0, \ldots, n)$ and $b_{q}(q=0, \ldots, m)$ are constant coefficients, $\alpha_{p}(p=0, \ldots, n)$ and $\beta_{q}(q=$ $0, \ldots, m)$ are real differential orders, $L$ is a time delay, $y(t)$ and $u(t)$ are the output and input of each process, respectively.

In order to approximate fractional differentiator, $s^{\alpha}$, where $(\alpha \in R)$ computationally, many functions and definitions are available (Petráš, 2011). Among a few of them are being commonly used in engineering applications, for example Riemann-Liouville (R-L), Caputo, Weyl and Grunwald-Letnikov (G-L) definitions. In our implantation of algorithm an R-L definition is used based on Euler's Gamma function.

\subsection{Identification and TITO process}

The identification structure of TITO process is depicted in Figure 1, where $u_{1}, u_{2}$ are inputs and $y_{1}$, $y_{2}$ are outputs of process. It comprises of a symmetrical relay pair associated with parallel controllers in closed-loop configuration to generate inputs for TITO process. The purpose of using relay is only to induce sustained oscillations termed as limit cycles to provide adequate input to excite TITO process dynamics

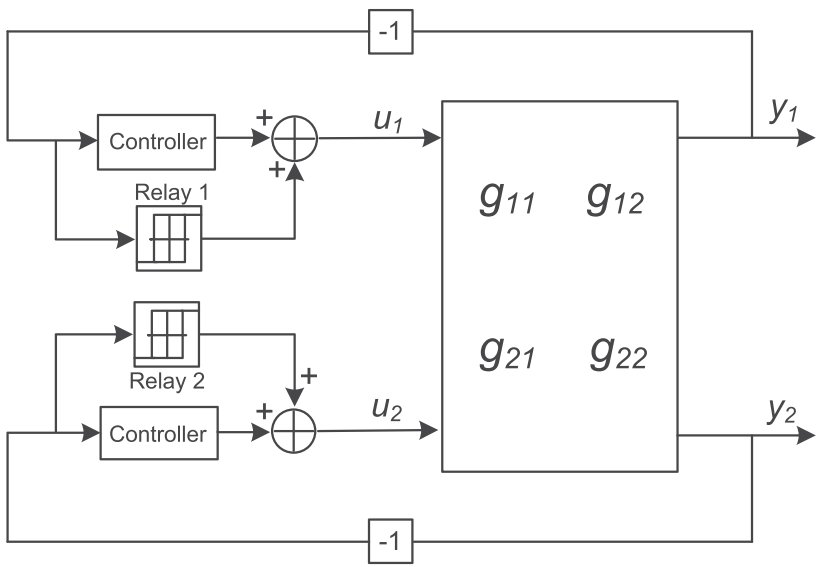

Figure 1. Proposed identification structure for TITO process. at desired level. Although relays are employed for identification experiment, it is not essential to wait for convergence unlike normal conventional approaches on relay autotuning. Any step input transient data or first two cycles from oscillations are sufficient which implicitly makes identification faster without compromising the overall efficiency.

In following we discuss the proposed identification steps to get fractional first-order time delay (FFOTD) models of a TITO process. It accomplishes identification in two sequential steps. In first step, relay- 1 is connected in the first loop (while a relay- 2 remains open in the second loop) until the output is reached at $t=t_{1}$. The input and output data are collected and termed as $\left[u_{1}^{1}, u_{2}^{1}\right]$ and $\left[y_{1}^{1}, y_{2}^{1}\right]$, respectively. Similarly, in second step the data $\left[u_{1}^{2}, u_{2}^{2}\right]$ and $\left[y_{1}^{2}, y_{2}^{2}\right]$ are collected with relay- 2 until the output is reached again at $t=t_{2}$ (while a relay- 1 is removed in first loop). After two sequential steps of relay experiment, the process is activated with inputs as shown in Figure 2 for various time periods. As show in figure, inputs for TITO process where time slot $t_{0}$ to $t_{1}$ shows first step and $t_{1}$ to $t_{2}$ shows second step of experiment.

As per TITO process transfer function in (1), the input-output relationship can be written as,

$$
\begin{aligned}
y_{1}^{i} & =g_{11} u_{1}^{i}+g_{12} u_{2}^{i} \\
y_{2}^{i} & =g_{21} u_{1}^{i}+g_{22} u_{2}^{i}
\end{aligned}
$$

where $i$ denotes first or second step data, here $i=1$ or 2. Although this experiment is conducted in a closed loop, the correlation of $u$ and $y$ is negligible when the input $u_{1}^{i}$ or $u_{2}^{i}$ is kept constant except the time of relay

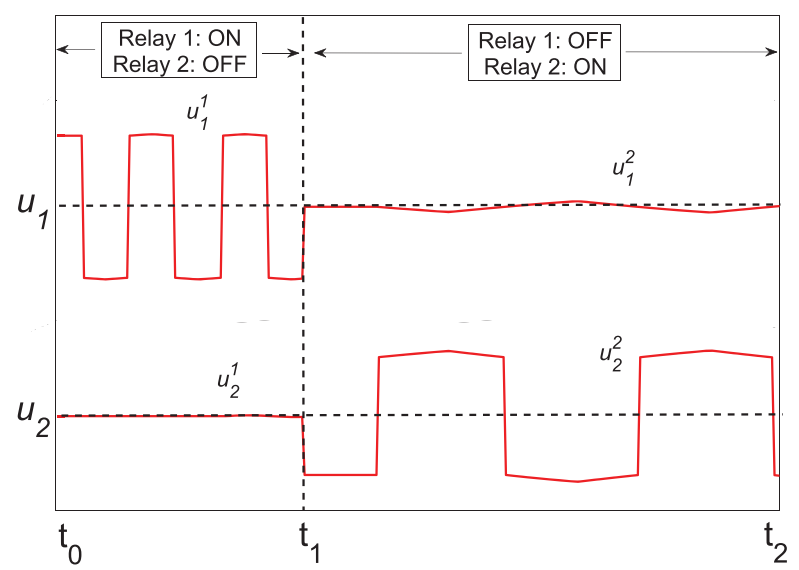

Figure 2. Input signals from sequential steps during identification. 

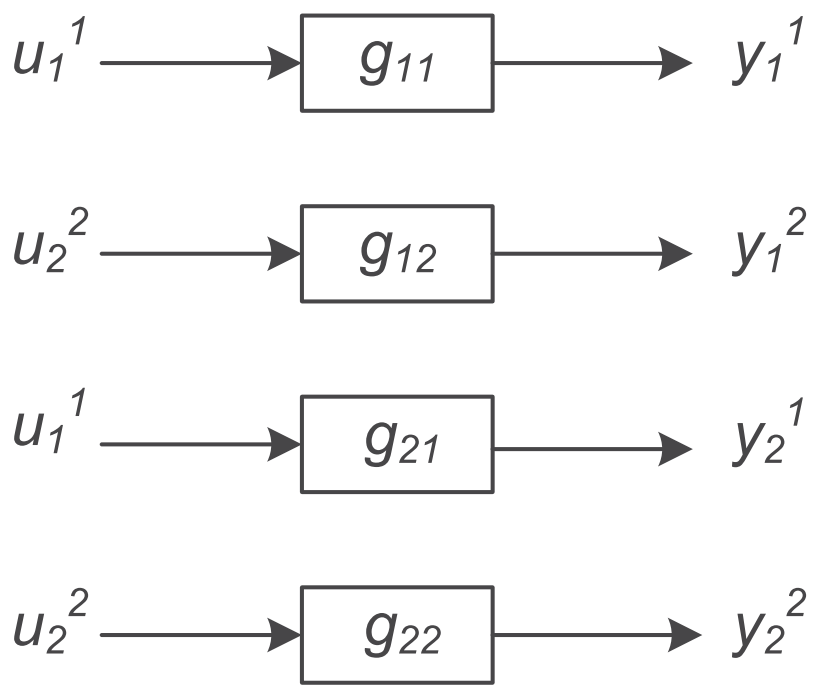

Figure 3. TITO process to four openloop SISO processes.

turns off. The inputs, $u_{2}^{1}$ and $u_{1}^{2}$ are sufficiently small to be ignored for identification purposes. Such condition in relay experiments provide an open-loop behaviour in multivariable processes. Therefore, it is possible to rewrite $y_{1}^{1}=g_{11} u_{1}^{1}, y_{2}^{1}=g_{21} u_{1}^{1}, y_{1}^{2}=g_{12} u_{2}^{2}$ and $y_{2}^{2}=$ $g_{22} u_{2}^{2}$ as shown in Figure 3.

As per more desirable features of relay feedback test described by Tan et al. (2006), a preload symmetrical relay is used here in data collection. A process is perturbed in the closed-loop through a relay test together with a proportional controller in parallel. The output starts oscillations nearby steady state value and as per allowable process variable swings. This feature is adopted in obtaining process input and output data. In our experiment, appropriate values of relay height and gain are anticipated and applied to accomplish precise identification.

\section{Proposed technique}

In the preceding section, the method is presented how the sequential relay tests are applied to the TITO process and the data $\left\{u_{1}^{1}(t), u_{2}^{2}(t)\right\}$ and $\left\{y_{1}^{1}(t), y_{1}^{2}(t), y_{2}^{1}(t)\right.$, $\left.y_{2}^{2}(t)\right\}$ are generated. After the responses are recorded, the main objective is to estimate four individual openloop transfer functions, namely FFOTD model. Firstly, it is necessary to tackle the fractional-order differential equations carefully due to fractional-order transfer functions. Obviously, a fractional-order derivatives can not be tackled as simply as integer-order. In this method, we have employed the operational matrix based on Haar wavelets to solve the problem. In following, first an operational matrix for delayed Haar wavelet is obtained due to time delay in process and then we convert the complex fractional differential equations into simple algebraic expressions for easy identification procedure.

\subsection{Operational matrix of fractional order integration for delayed Haar wavelet}

Haar wavelets are the elementary functions of wavelet family. Also, they are the simplest and oldest orthonormal wavelet with compact support (Jiwari, 2012). These piecewise constant functions are employed in proposed technique, owing to their excellent properties including high accuracy, mathematical simplicity, computationally faster, noise immunity and their ease of implementation with other standard algorithms (Kothari et al., 2018a). Using Haar wavelets, an arbitrary function $x(t) \in L^{2}\left[0, T_{f}\right]$ can be written for the first $M$ terms as (Jiwari, 2012; Li et al., 2015),

$$
x(t)=\sum_{i=0}^{M-1} c_{i} h_{i}(t)=C_{M}^{T} H_{M}(t)
$$

Here, the Haar coefficient vector $C_{M} \triangleq\left[c_{0}, c_{1}, \ldots\right.$, $\left.c_{M-1}\right]^{T}$ and Haar function vector $H_{M}(t) \triangleq\left[h_{0}(t)\right.$, $\left.h_{1}(t), \ldots, h_{M-1}(t)\right]^{T}$.

In (5), function $x(t)$ with infinite series is approximated for first $M$ terms. For practical processes, it is not possible to consider infinite datapoints or entire collected dataset in order to maintain computational efforts (Y. Li et al., 2017). Therefore appropriate datalength needs to be chosen for optimal accuracy and speed. In our case, Haar wavelets datalength $M$ is always power of 2 and commonly preferable values are $M=128$ and $M=256$ to meet the accuracy of identification.

The $M$-square Haar matrix $\Psi_{M \times M}$ can be derived as (Li et al., 2015),

$$
\begin{aligned}
\Psi_{M \times M} \triangleq & {\left[H_{M}\left(\frac{1}{2 M} T_{f}\right) H_{M}\left(\frac{3}{2 M} T_{f}\right) \ldots\right.} \\
& \left.H_{M}\left(\frac{2 M-1}{2 M} T_{f}\right)\right]
\end{aligned}
$$

The generalised operational matrix of fractional order integration (FOI) can be derived using basic orthogonal piecewise constant block pulse functions. 
The analytical expression of operational matrix of FOI for any absolutely integrable function can be derived using generalised operational matrix of FOI. The FOI of block pulse functions can be written as,

$$
\left(\mathscr{I}^{\alpha} \Phi_{M}\right)(t) \approx F_{M \times M}^{\alpha} \Phi_{M}(t)
$$

where $M$ shows number of elementary functions, $\Phi_{M}(t)$ denotes block pulse functions over the time interval $\left[0, T_{f}\right]$ and $F_{M \times M}^{\alpha}$ is called the generalised operational matrix of fractional integral given by

$F_{M \times M}^{\alpha}=\left(\frac{T_{f}}{M}\right)^{\alpha} \frac{1}{\Gamma(\alpha+2)}\left(\begin{array}{ccccc}1 & f_{1} & f_{2} & \ldots & f_{M-1} \\ 0 & 1 & f_{1} & \ldots & f_{M-2} \\ 0 & 0 & 1 & \ldots & f_{M-3} \\ 0 & 0 & 0 & \ddots & \vdots \\ 0 & 0 & 0 & \ldots & 1\end{array}\right)$

where $f_{z}=(z+1)^{\alpha+1}-2(z)^{\alpha+1}+(z-1)^{\alpha+1}$ and $z=1,2,3, \ldots, M-1$.

Haar wavelet operational matrix of integration $P_{M \times M}^{\alpha}$ can be obtained using the generalised operational matrix of integration $F_{M \times M}^{\alpha}$. Let the fractional integration of the $H_{M}(t)$ is expressed by

$$
\left(\mathscr{I}^{\alpha} H_{M}\right)(t) \approx P_{M \times M}^{\alpha} H_{M}(t)
$$

where square matrix $P_{M \times M}^{\alpha}$ denotes Haar wavelet operational matrix of FOI. Haar wavelet functions can be expanded into an $M$-terms block pulse functions, as they are piecewise constant, by

$$
H_{M}(t)=\Psi_{M \times M} \Phi_{M}(t)
$$

Therefore, using (7) and (10), one can obtain

$$
\left(\mathscr{I}^{\alpha} H_{M}\right)(t) \approx \Psi_{M \times M} F_{M \times M}^{\alpha} \Phi_{M}(t)
$$

We know, an orthogonal matrix is always invertible and its inverse is same as its transpose. As the $M$ square Haar matrix $\Psi_{M \times M}$ is an orthogonal matrix so its inverse $\Psi_{M \times M}^{-1}$ always exists.

Comparing (9) and (11), the analytical expression of Haar wavelet operational matrix of fractional integration is obtained as (Li et al., 2015),

$$
P_{M \times M}^{\alpha}=\Psi_{M \times M} F_{M \times M}^{\alpha} \Psi_{M \times M}^{-1}
$$

In case when input is delayed by the value $L$, a shifted block pulse functions shall be given as $\Phi_{M}(t-L)$ and written by Tang et al. (2017),

$$
\Phi_{M}(t-L)=D \Phi_{M}(t)
$$

where $D$ is the $M$-square generalised delay operational matrix and obtained as

$$
D=\left[\begin{array}{ccccccc}
0 & \cdots & 0 & 1 & 0 & \cdots & 0 \\
0 & \cdots & 0 & 0 & 1 & \cdots & 0 \\
\vdots & & \vdots & \vdots & \vdots & \ddots & \vdots \\
0 & \cdots & 0 & 0 & 0 & \cdots & 1 \\
0 & \cdots & 0 & 0 & 0 & \cdots & 0 \\
\vdots & & \vdots & \vdots & \vdots & & \vdots \\
0 & \cdots & 0 & 0 & 0 & \cdots & 0
\end{array}\right]_{(M \times M)}
$$

Similarly, in order to derive the delay operational matrix of Haar wavelet, we consider the delayed Haar wavelet function as $H_{M}(t-L)$. It can be characterised as

$$
H_{M}(t-L)=D_{H} H_{M}(t)
$$

where $t>L, 0 \leqslant t \leqslant T_{f}$ and $D_{H}$ is the $M$-square delay operational matrix of the Haar wavelet. Using (10), $H_{M}(t-L)$ can be expressed as,

$$
H_{M}(t-L)=\Psi_{M \times M} \Phi_{M}(t-L)
$$

Comparing (15) and (16) gives,

$$
D_{H} H_{M}(t)=\Psi_{M \times M} \Phi_{M}(t-L)
$$

Now, by substituting values of (10) and (13) into (17), one can obtain

$$
D_{H} \Psi_{M \times M} \Phi_{M}(t)=\Psi_{M \times M} D \Phi_{M}(t)
$$

Above expression gives the Haar wavelet delay operational matrix $D_{H}$ as (Kothari et al., 2018a)

$$
D_{H}=\Psi_{M \times M} D \Psi_{M \times M}^{-1}
$$

At the end after performing the R-L definition of fractional integration on $H_{M}(t-L)$ using (9) and (15), following equation is obtained.

$$
\begin{aligned}
\left(\mathscr{I}^{\alpha} H_{M}\right)(t-L) & =D_{H}\left(\mathscr{I}^{\alpha} H_{M}\right)(t) \\
& =D_{H} P_{M \times M}^{\alpha} H_{M}(t)
\end{aligned}
$$

Thus, $\left(\mathscr{I}^{\alpha} H_{M}\right)(t-L)$ is thereby obtained simply by matrix multiplication of $H_{M}(t)$ with $P_{M \times M}^{\alpha}$ and $D_{H}$. It is worth noting that the proposed technique using operational matrices is straightforward and potentially omits the complex calculation of fractional-order equations. 


\subsection{Modeling of fractional order time delay process}

As unveiled in previous section, the TITO process can be observed as four stable independent open loop processes if the proposed test is adopted. Therefore, the generalised transfer function can be given using a single pole model for each process as,

$$
g_{i j}=\frac{Y_{i}^{j}(s)}{U_{j}^{j}(s)}=\frac{K_{i j}}{T_{i j} s^{\alpha_{i j}}+1} \mathrm{e}^{-L_{i j} s}
$$

where $K_{i j}(i, j=1,2)$ are process gain, $T_{i j}(i, j=1,2)$ are time constants, $L_{i j}(i, j=1,2)$ are time delays, $\alpha_{i j}(i, j=1,2)\left(\alpha_{i j} \in R\right)$ are real orders of differentiation. Now expressing (21) in time domain,

$$
T_{i j} \mathscr{D}^{\alpha_{i j}} y_{i}^{j}(t)+y_{i}^{j}(t)=K_{i j} u_{j}^{j}(t-L)
$$

After integrating with the fractional order of $\alpha_{i j}$, one can obtain,

$$
T_{i j} \mathscr{I}^{\alpha_{i j}} \mathscr{D}^{\alpha_{i j}} y_{i}^{j}(t)+\mathscr{I}^{\alpha_{i j}} y_{i}^{j}(t)=\mathscr{I}^{\alpha_{i j}} K_{i j} u_{j}^{j}(t-L)
$$

Simplification and rearrangement gives,

$$
T_{i j} y_{i}^{j}(t)+\mathscr{I}^{\alpha_{i j}} y_{i}^{j}(t)=K_{i j} \mathscr{I}^{\alpha_{i j}} u_{j}^{j}(t-L)
$$

Now, suppose the input and output of the process is expressed in terms of Haar wavelets as shown in Section 3.1, we can write

$$
\begin{aligned}
& y_{i}^{j}(t)=Y_{i}^{j}{ }^{T} H_{M}(t) \\
& u_{j}^{j}(t-L)=U_{j}^{j}{ }^{j} H_{M}(t-L)
\end{aligned}
$$

where $Y_{i}^{j^{T}}=\left[y_{i 1}^{j}, y_{i 2}^{j}, \ldots, y_{i M}^{j}\right]$ and $U_{j}^{j T}=\left[u_{j_{1}}^{j}, u_{j_{2}}^{j}\right.$, $\left.\ldots, u_{j_{M}}^{j}\right]$ and superscript $T$ denotes the transpose. Now substituting the values of (25) and (26) into (24), one can obtain,

$$
\begin{gathered}
T_{i j} Y_{i}^{j^{T}} H_{M}(t)+Y_{i}^{j} \mathscr{I}^{\alpha_{i j}} H_{M}(t) \\
=K_{i j} U_{j}^{j} \mathscr{I}^{\alpha_{i j}} H_{M}(t-L)
\end{gathered}
$$

In aforementioned equation, the integration of input and output can be given as, $\mathscr{I}^{\alpha_{i j}}\left(H_{M}(t)\right)=P^{\alpha_{i j}} H_{M}(t)$ and $\mathscr{I}^{\alpha_{i j}}\left(H_{M}(t-L)\right)=D_{H} P^{\alpha_{i j}} H_{M}(t)$ as obtained in
Section 3.1, Therefore, (27) can be rewritten as,

$$
T_{i j}{ }_{i}^{j^{j}} H_{M}(t)+Y_{i}^{j}{ }^{T} P^{\alpha_{i j}} H_{M}(t)=K_{i j} U_{j}^{j^{T}} D_{H} P^{\alpha_{i j}} H_{M}(t)
$$

After simplification, one can obtain,

$$
Y_{i}^{j^{T}}=U_{j}^{j^{T}} D_{H}\left(K_{i j} P^{\alpha_{i j}}\right)\left(T_{i j} I_{M \times M}+P^{\alpha_{i j}}\right)^{-1}
$$

where $I_{M \times M}$ represents identity matrix. Finally, an expression for output $y(t)$ can be attained as,

$$
y_{i}^{j}(t)=U_{j}^{j}{ }^{T} D_{H}\left(K_{i j} P^{\alpha_{i j}}\right)\left(T_{i j} I_{M \times M}+P^{\alpha_{i j}}\right)^{-1} H_{M}(t)
$$

This expression helps to calculate each independent SISO process output from estimated values of model parameters. Now in order to identify four transfer functions as TITO process, the appropriate dataset is produced as discussed in Section 2.1. The time response datasets with $M$ number of samples $\left\{u_{\text {data }}(k), y_{\text {data }}(k)\right\}_{k=1, \ldots M}$ are collected after relay experiment.

It is well-known in parameter identification to minimise corresponding objective function. The timemoment weighted integral performance criterion has been widely used as an objective function. It employs the integral of squared-time-weighted-error (ISTE) criterion to estimate the system parameters and can be written as per our estimation values as

$$
J_{I S T E}=\min _{\rho} \sum_{k=1}^{M}\left[k\left(y(k)-y_{\text {data }}(k)\right)\right]^{2}
$$

where $\rho$ is the vector of unknown parameters ( $K, T, \alpha, L), y(k)$ is the time domain response of (30) calculated using $\rho, y_{\text {data }}(k)$ is the collected actual output to fit to $y(k)$. In (31), $y(k)$ and $y_{\text {data }}(k)$ are the simulated response and collected response data at time $t_{k}$, respectively and $M$ is the total number of samples. This routine aims to find the model parameters that would ideally reduce the ISTE as minimum as possible. The MATLAB function $\mathrm{fsolve}$ is used to calculate the best estimated parameters which satisfies the above objective function in (31).

In our analysis the time and frequency domain errors have been calculated for the result comparison. This is because for some cases, even though result gives the close approximations in one domain but deviates too far away from the real system response in other domain. The time domain identification error $\varepsilon_{t}$ is 
calculated by,

$$
\varepsilon_{t}=\frac{1}{M} \sum_{t=1}^{M}\left[y_{\text {data }}(t)-y(t)\right]^{2}
$$

where $y_{\text {data }}$ is the actual and $y$ is the output from the estimated model with same input excitation. Similarly, the frequency domain error $\varepsilon_{f}$ can be calculated as the worst-case error by

$$
\varepsilon_{f}=\max _{\omega \in\left[0, \omega_{c}\right]}\left\{\left|\frac{\hat{g}(j \omega)-g(j \omega)}{g(j \omega)}\right| \times 100 \%\right\}
$$

where frequency responses $g$ and $\hat{g}$ are from true and estimated models respectively. The frequency range $\left[0, \omega_{c}\right]$ was selected to evaluate where $\angle g\left(j \omega_{c}\right)=$ $-\pi$.

\section{Verification and analysis}

In this section, three different types of examples from the literature have been chosen to demonstrate the effectiveness of the proposed identification method. First, the well-known benchmark example of Wood and Berry binary distillation column plant studied in literature (Berner, Soltesz, Hägglund, et al., 2017; S.Y. Li et al., 2005b; Nema \& Padhy, 2015; Tsay, 2011) is considered. This TITO process has strong loop interaction, sufficiently large time delays and adequate gain values. Second example is considered with higher integer-order dynamics from Nema and Padhy (2015), Tsay (2011) and corresponding TITO process is approximated using proposed FFOTD models. Third example is chosen with higher-order fractional derivatives from Xue and Li (2018). The obtained results are compared with recent methods for TITO processes namely, Autotuner identification of TITO process (AIT) (Berner, Soltesz, Hägglund, et al., 2017), State-space based TITO identification (SST) (Nema \& Padhy, 2015), Automatic tuning method for TITO process (ATT) (Tsay, 2011) and Effective decentralised TITO process identification (EDT) (S.-Y. Li et al., 2005b).

The process dynamics for considered examples are given as follows.

$G_{1}(s)=\left[\begin{array}{cc}\frac{12.8}{1+16.7 s} \mathrm{e}^{-s} & \frac{-18.9}{1+21 s} \mathrm{e}^{-3 s} \\ \frac{6.6}{1+10.9 s} \mathrm{e}^{-7 s} & \frac{-19.4}{1+14.4 s} \mathrm{e}^{-3 s}\end{array}\right]$
$G_{2}(s)=\left[\begin{array}{cc}\frac{0.5}{(0.1 s+1)^{2}(0.2 s+1)^{2}} & \frac{-1}{(0.1 s+1)(0.2 s+1)^{2}} \\ \frac{1}{(0.1 s+1)(0.2 s+1)^{2}} & \frac{2.4}{(0.1 s+1)(0.2 s+1)^{2}(0.5 s+1)}\end{array}\right]$

$G_{3}(s)=\left[\begin{array}{cc}\frac{1}{1.35 s^{1.2}+2.3 s^{0.9}+1} \mathrm{e}^{-0.2 s} & \frac{2}{4.13 s^{0.7}+1} \mathrm{e}^{-0.2 s} \\ \frac{1}{0.52 s^{1.5}+2.03 s^{0.7}+1} & \frac{-1}{3.8 s^{0.8}+1} \mathrm{e}^{-0.5 s}\end{array}\right]$

For all chosen examples, we have collected first and second step data for output $y_{1}$ and $y_{2}$ and corresponding four independent transfer functions for each TITO process have been approximated. Table 1 shows results from the proposed method and other reported in literature. The value of each parameter is obtained by calculating its mean value of total trials. The results are also compared using time domain outputs from actual and estimated models as shown in Figures 4-6 for $G_{1}$, Figures 8-10 for $G_{2}$ and Figures 12 and 13

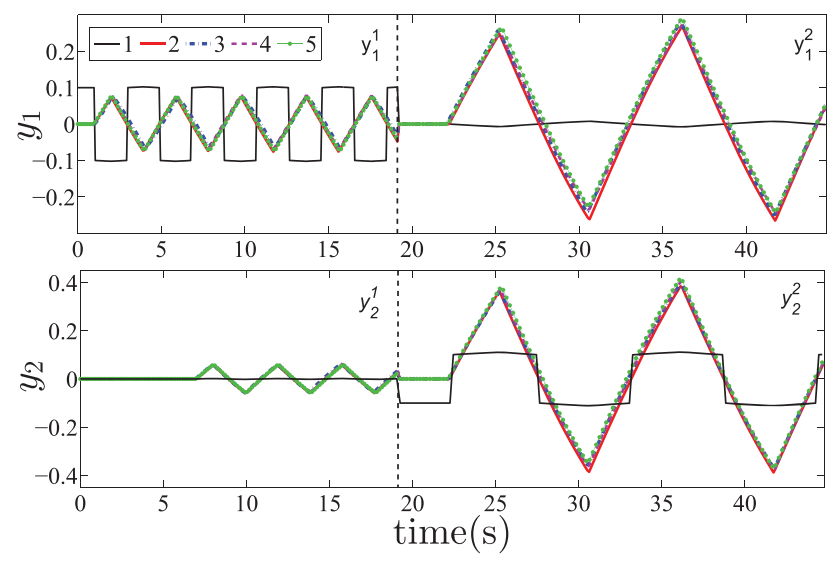

Figure 4. Time responses for $G_{1}$ : (1) input (2) actual, (3) proposed model, (4) AIT model and (5) SST model.
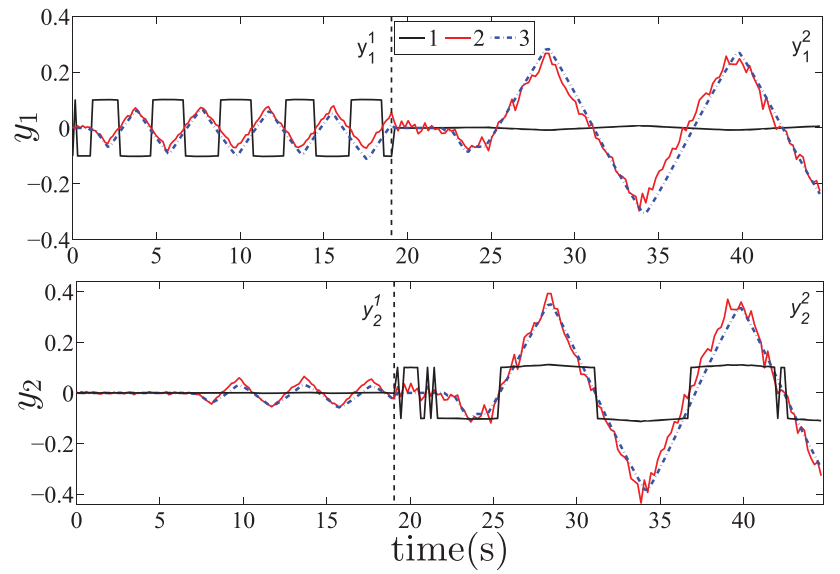

Figure 5. Time responses for $G_{1}$ with $\mathrm{SNR}=20 \mathrm{~dB}$ : (1) input (2) actual and (3) proposed model. 
Table 1. Identification results.

\begin{tabular}{|c|c|c|c|c|c|c|c|}
\hline \multirow{2}{*}{$\begin{array}{l}\text { Process } \\
G_{1}(s)\end{array}$} & \multirow{2}{*}{$\begin{array}{l}\text { Method } \\
\text { Proposed }\end{array}$} & \multicolumn{2}{|c|}{ Identified models } & \multicolumn{2}{|c|}{ Error $\varepsilon_{t}\left[\begin{array}{ll}y_{1} & y_{2}\end{array}\right]$} & \multicolumn{2}{|c|}{ Error $\varepsilon_{f}$} \\
\hline & & {$\left[\begin{array}{l}\frac{12.82}{16.68 s^{0.98}+1} \mathrm{e}^{-1.02 s} \\
\frac{6.71}{11.16 s^{0.97}+1} \mathrm{e}^{-6.92 s}\end{array}\right.$} & $\begin{array}{l}\frac{-18.84}{20.94 s^{1.01}+1} \mathrm{e}^{-2.87 \mathrm{~s}} \\
\frac{-19.29}{14.68 s^{0.99}+1} \mathrm{e}^{-2.96 s}\end{array}$ & {$\left[3.73 \times 10^{-4}\right.$} & $\left.4.13 \times 10^{-4}\right]$ & {$\left[\begin{array}{l}2.30 \\
2.10\end{array}\right.$} & $\left.\begin{array}{l}1.28 \\
1.55\end{array}\right]$ \\
\hline & Proposed $(\mathrm{SNR}=20$ ) & {$\left[\begin{array}{l}\frac{12.57}{15.29 s^{0.97}+1} \mathrm{e}^{-0.99 s} \\
\frac{5.90}{12.06 \mathrm{~s}^{1.16}+1} \mathrm{e}^{-6.89 \mathrm{~s}}\end{array}\right.$} & $\left.\begin{array}{l}\frac{-19.01}{19.88 s^{1.06}+1} \mathrm{e}^{-2.89 \mathrm{~s}} \\
\frac{-19.02}{15.96 \mathrm{~s}^{1.07}+1} \mathrm{e}^{-2.89 \mathrm{~s}}\end{array}\right]$ & {$\left[6.98 \times 10^{-4}\right.$} & $\left.7.05 \times 10^{-4}\right]$ & {$\left[\begin{array}{l}2.66 \\
6.70\end{array}\right.$} & $\left.\begin{array}{c}12.16 \\
3.83\end{array}\right]$ \\
\hline & Proposed $(\mathrm{SNR}=40)$ & {$\left[\begin{array}{l}\frac{12.49}{15.4 s^{0.98}+1} \mathrm{e}^{-0.99 s} \\
\frac{6.14}{9.43 s^{0.98}+1} \mathrm{e}^{-7.09 \mathrm{~s}}\end{array}\right.$} & $\left.\begin{array}{l}\frac{-19.46}{22.61 s^{1.01}+1} \mathrm{e}^{-2.99 s} \\
\frac{-19.15}{15.46 \mathrm{~s}^{1.06}+1} \mathrm{e}^{-2.89 \mathrm{~s}}\end{array}\right]$ & {$\left[1.07 \times 10^{-4}\right.$} & $\left.4.95 \times 10^{-4}\right]$ & {$\left[\begin{array}{l}2.34 \\
4.60\end{array}\right.$} & $\left.\begin{array}{l}1.96 \\
4.44\end{array}\right]$ \\
\hline & AIT & {$\left[\begin{array}{l}\frac{15.9}{21.0 s+1} \mathrm{e}^{-1.03 s} \\
\frac{5.84}{9.62 s+1} \mathrm{e}^{-7.02 s}\end{array}\right.$} & $\left.\begin{array}{l}\frac{-18.2}{20.7 s+1} \mathrm{e}^{-3.00 s} \\
\frac{-20.2}{15.1 s+1} \mathrm{e}^{-2.99 s}\end{array}\right]$ & {$\left[5.17 \times 10^{-4}\right.$} & $\left.4.33 \times 10^{-4}\right]$ & {$\left[\begin{array}{c}12.92 \\
8.18\end{array}\right.$} & $\left.\begin{array}{l}3.22 \\
2.35\end{array}\right]$ \\
\hline & EDT & {$\left[\begin{array}{l}\frac{12.79}{16.62 s+1} e^{-1.00 s} \\
\frac{6.59}{10.92 s+1} e^{-6.98 s}\end{array}\right.$} & $\left.\begin{array}{l}\frac{-18.90}{21.00 s+1} \mathrm{e}^{-3.01 s} \\
\frac{-19.39}{14.43 s+1} \mathrm{e}^{-3.01 \mathrm{~s}}\end{array}\right]$ & {$\left[5.27 \times 10^{-4}\right.$} & $\left.6.49 \times 10^{-4}\right]$ & {$\left[\begin{array}{c}0.17 \\
0.134\end{array}\right.$} & $\left.\begin{array}{l}0.094 \\
0.189\end{array}\right]$ \\
\hline & ATT & {$\left[\begin{array}{l}\frac{12.8}{16.69 s+1} \mathrm{e}^{-1.01 s} \\
\frac{6.6}{10.89 s+1} \mathrm{e}^{-7.01 s}\end{array}\right.$} & $\left.\begin{array}{l}\frac{-18.9}{21.01 s+1} \mathrm{e}^{-3.01 s} \\
\frac{-19.4}{14.41 s+1} \mathrm{e}^{-3.01 \mathrm{~s}}\end{array}\right]$ & {$\left[5.28 \times 10^{-4}\right.$} & $\left.6.50 \times 10^{-4}\right]$ & {$\left[\begin{array}{l}0.20 \\
0.17\end{array}\right.$} & $\left.\begin{array}{c}0.17 \\
0.212\end{array}\right]$ \\
\hline & SST & {$\left[\begin{array}{l}\frac{12.79}{16.601 s+1} e^{-1.00 s} \\
\frac{6.6}{10.90 s+1} e^{-6.99 s}\end{array}\right.$} & $\left.\begin{array}{l}\frac{-18.90}{21.0 s+1} e^{-2.99 s} \\
\frac{-19.39}{14.44 s+1} e^{-3.01 s}\end{array}\right]$ & {$\left[3.06 \times 10^{-4}\right.$} & $\left.4.08 \times 10^{-4}\right]$ & {$\left[\begin{array}{l}0.27 \\
0.04\end{array}\right.$} & $\left.\begin{array}{l}0.09 \\
0.15\end{array}\right]$ \\
\hline$G_{2}(s)$ & Proposed & {$\left[\begin{array}{l}\frac{0.48}{0.44 s^{1.11}+1} \mathrm{e}^{-0.22 s} \\
\frac{1.02}{0.50 s^{1.05}+1} \mathrm{e}^{-0.19 s}\end{array}\right.$} & $\left.\begin{array}{l}\frac{-0.97}{0.35 s^{1.15}+1} \mathrm{e}^{-0.09 s} \\
\frac{2.52}{0.95 s^{1.1}+1} \mathrm{e}^{-0.39 s}\end{array}\right]$ & {$\left[4.10 \times 10^{-3}\right.$} & $\left.1.34 \times 10^{-2}\right]$ & {$\left[\begin{array}{c}7.36 \\
20.12\end{array}\right.$} & $\left.\begin{array}{c}9.41 \\
19.71\end{array}\right]$ \\
\hline & Proposed $(\mathrm{SNR}=20)$ & {$\left[\begin{array}{l}\frac{0.46}{0.46 s^{1.14}+1} e^{-0.25 s} \\
\frac{1.02}{0.49 s^{1.04}+1} e^{-0.19 s}\end{array}\right.$} & $\left.\begin{array}{l}\frac{-0.97}{0.35 s^{1.16}+1} \mathrm{e}^{-0.12 s} \\
\frac{2.51}{0.95 s^{1.08}+1} \mathrm{e}^{-0.39 \mathrm{~s}}\end{array}\right]$ & {$\left[3.04 \times 10^{-3}\right.$} & $\left.1.53 \times 10^{-2}\right]$ & {$\left[\begin{array}{l}18.78 \\
21.04\end{array}\right.$} & $\left.\begin{array}{c}1.83 \\
19.85\end{array}\right]$ \\
\hline & Proposed $(\mathrm{SNR}=40)$ & {$\left[\begin{array}{l}\frac{0.46}{0.46 s^{1.13}+1} \mathrm{e}^{-0.25 s} \\
\frac{1.02}{0.49 s^{1.04}+1} \mathrm{e}^{-0.2 s}\end{array}\right.$} & $\left.\begin{array}{l}\frac{-0.97}{0.34 s^{1.16}+1} \mathrm{e}^{-0.12 s} \\
\frac{2.51}{0.95 s^{1.08}+1} \mathrm{e}^{-0.39 \mathrm{~s}}\end{array}\right]$ & {$\left[9.67 \times 10^{-4}\right.$} & $\left.1.20 \times 10^{-2}\right]$ & {$\left[\begin{array}{l}17.98 \\
21.04\end{array}\right.$} & $\left.\begin{array}{c}2.72 \\
19.85\end{array}\right]$ \\
\hline & SST & {$\left[\begin{array}{l}\frac{0.5}{0.69 s+1} e^{-3.8 s} \\
\frac{1}{0.31 s+1} e^{-0.126 s}\end{array}\right.$} & $\left.\begin{array}{l}\frac{-1}{0.31 s+1} \mathrm{e}^{-0.126 s} \\
\frac{2.4}{0.40 s+1} \mathrm{e}^{-0.2 s}\end{array}\right]$ & {$\left[1.58 \times 10^{-2}\right.$} & $\left.3.49 \times 10^{-1}\right]$ & {$\left[\begin{array}{l}45.13 \\
21.20\end{array}\right.$} & $\left.\begin{array}{l}21.20 \\
57.35\end{array}\right]$ \\
\hline & ATT & {$\left[\begin{array}{l}\frac{0.5}{0.32 s+1} \mathrm{e}^{-2.79 s} \\
\frac{1}{0.305 s+1} \mathrm{e}^{-0.196 s}\end{array}\right.$} & $\left.\begin{array}{l}\frac{-1}{0.304 s+1} \mathrm{e}^{-0.196 s} \\
\frac{2.4}{0.597 s+1} \mathrm{e}^{-0.409 s}\end{array}\right]$ & {$\left[9.95 \times 10^{-3}\right.$} & $\left.5.25 \times 10^{-2}\right]$ & {$\left[\begin{array}{l}9.75 \\
8.15\end{array}\right.$} & $\left.\begin{array}{l}8.30 \\
8.62\end{array}\right]$ \\
\hline$G_{3}(s)$ & Proposed & $\begin{array}{l}\frac{0.98}{3.99 s^{0.99}+1} \mathrm{e}^{-0.199 \mathrm{~s}} \\
\frac{0.97}{3.2 s^{0.78}+1} \mathrm{e}^{-8.54 \times 10^{-4} \mathrm{~s}}\end{array}$ & $\begin{array}{l}\frac{2.04}{4.10 s^{0.69}+1} \mathrm{e}^{-0.2 \mathrm{~s}} \\
\frac{-0.99}{3.79 s^{0.79}+1} \mathrm{e}^{-0.44 \mathrm{~s}}\end{array}$ & {$\left[6.87 \times 10^{-5}\right.$} & $\left.2.72 \times 10^{-4}\right]$ & {$\left[\begin{array}{c}6.88 \\
20.63\end{array}\right.$} & $\left.\begin{array}{l}2.69 \\
1.14\end{array}\right]$ \\
\hline & Proposed $(\mathrm{SNR}=20)$ & {$\left[\begin{array}{l}\frac{0.96}{3.95 s^{0.98}+1} \mathrm{e}^{-0.27 s} \\
\frac{0.98}{3.10 s^{0.78}+1} \mathrm{e}^{-0.09 s}\end{array}\right.$} & $\left.\begin{array}{l}\frac{2.08}{4.20 s^{0.78}+1} \mathrm{e}^{-0.19 s} \\
\frac{-0.98}{3.88 s^{0.76}+1} \mathrm{e}^{-0.52 \mathrm{~s}}\end{array}\right]$ & {$\left[8.07 \times 10^{-4}\right.$} & $\left.4.46 \times 10^{-4}\right]$ & {$\left[\begin{array}{c}6.71 \\
16.41\end{array}\right.$} & $\left.\begin{array}{l}9.23 \\
7.65\end{array}\right]$ \\
\hline & Proposed $(\mathrm{SNR}=40)$ & {$\left[\begin{array}{l}\frac{0.98}{3.99 s^{0.98}+1} \mathrm{e}^{-0.199 \mathrm{~s}} \\
\frac{0.97}{3.19 s^{0.78}+1} \mathrm{e}^{-0.07 s}\end{array}\right.$} & $\left.\begin{array}{l}\frac{2.04}{4.10 s^{0.69}+1} \mathrm{e}^{-0.199 \mathrm{~s}} \\
\frac{-0.99}{3.78 s^{0.79}+1} \mathrm{e}^{-0.49 s}\end{array}\right]$ & {$\left[8.16 \times 10^{-4}\right.$} & $\left.1.60 \times 10^{-4}\right]$ & {$\left[\begin{array}{c}6.71 \\
16.41\end{array}\right.$} & $\left.\begin{array}{l}9.23 \\
7.65\end{array}\right]$ \\
\hline
\end{tabular}



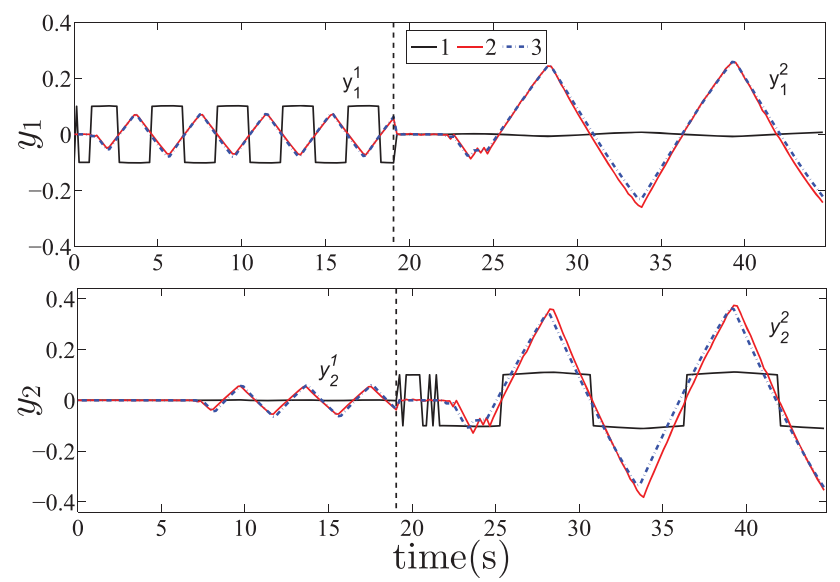

Figure 6. Time responses for $G_{1}$ with $\mathrm{SNR}=40 \mathrm{~dB}$ : (1) input (2) actual and (3) proposed model.

for $G_{3}$. Similarly, Nyquist plots for all examples have been compared in Figures 7, 11 and 14.

In order to confirm the usefulness of the proposed method under realistic conditions, the processes are also estimated in the face of measurement noise. Let the dataset be corrupted by Gaussian distributed random noise with signal-to-noise ratio (SNR) values of $20 \mathrm{~dB}$ and $40 \mathrm{~dB}$. It is interesting to note that the
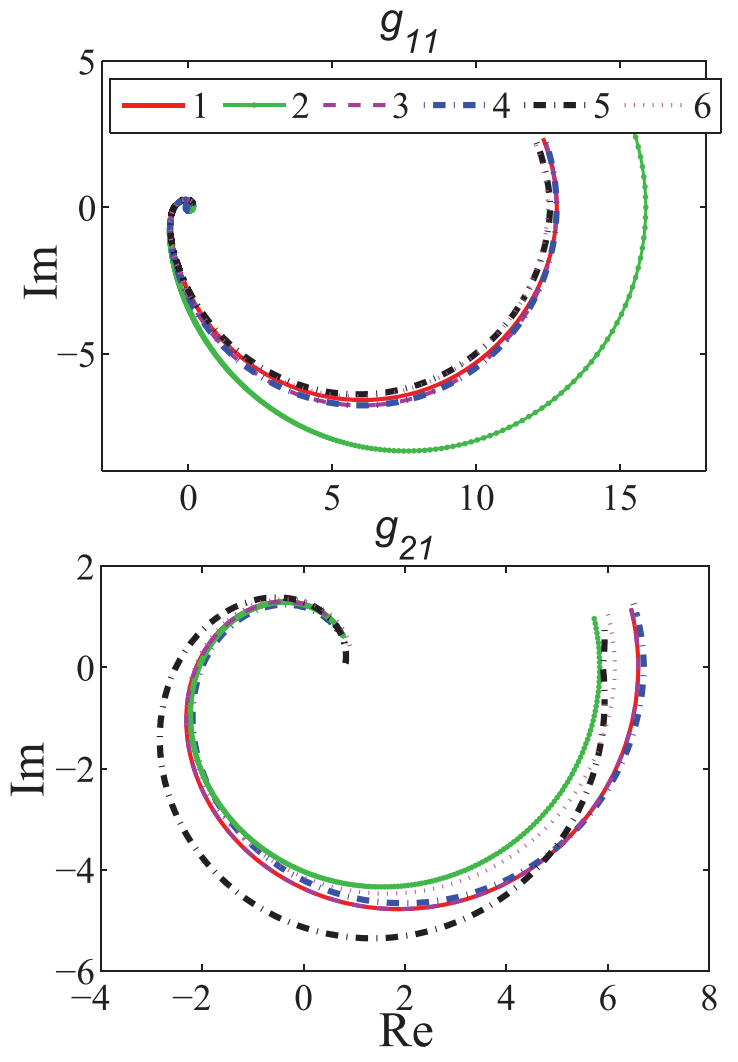
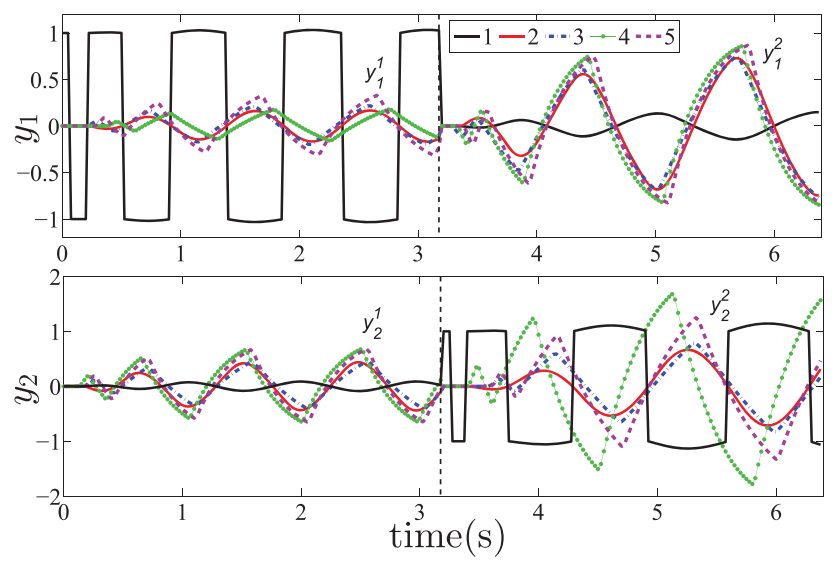

Figure 8. Time responses for $G_{2}$ : (1) input (2) actual, (3) proposed model (4) SST model and (5) ATT model.

proposed technique can handle the noisy environment well as proven from time responses. A key merit from the proposed Haar wavelet-based estimation is that it is not necessary to filter or clean the measured noisy data before applying identification procedure. Table 1 shows that the proposed idea can deal with noisy data and identify the process more accurately.
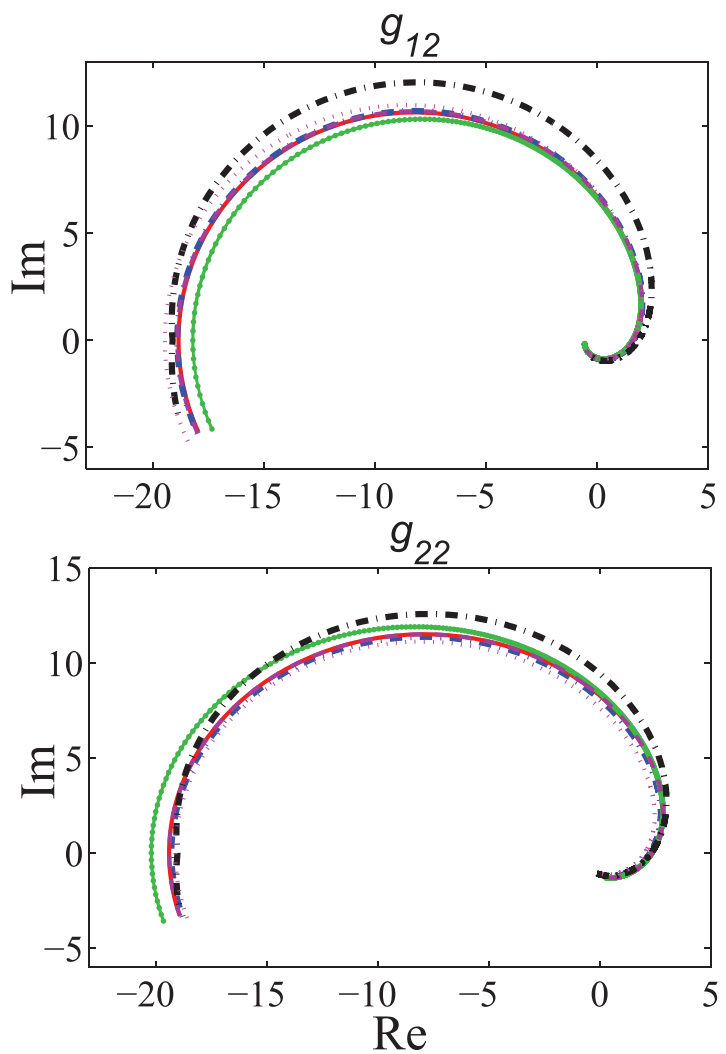

Figure 7. Nyquist plots for $G_{1}$ : (1) actual, (2) AIT model, (3) SST model, (4) proposed model, (5) proposed model (SNR = 20) and (6) proposed model $(\mathrm{SNR}=40)$. 

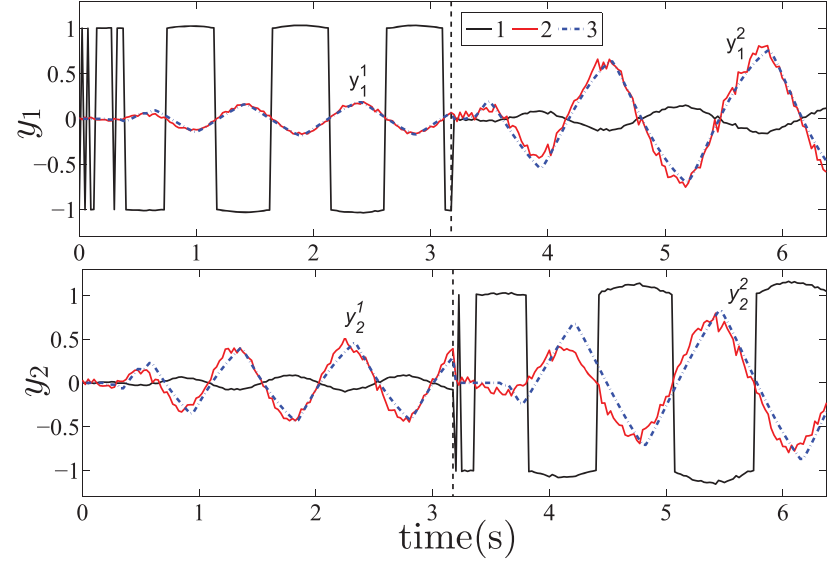

Figure 9. Time responses for $G_{2}$ with $\mathrm{SNR}=20$ : (1) actual and (2) proposed model.

First two examples have been estimated using fractional-order models and they are all near to their true processes. The other estimation methods are comparable to the one obtained in fractional transfer function. Additionally to note that there is no need to de-noise the corrupted signals to apply the identification algorithm. Further, for any fractional-order process, integer-order model can not give satisfactory accuracy. As shown for $G_{3}$ in Table 1, fractional
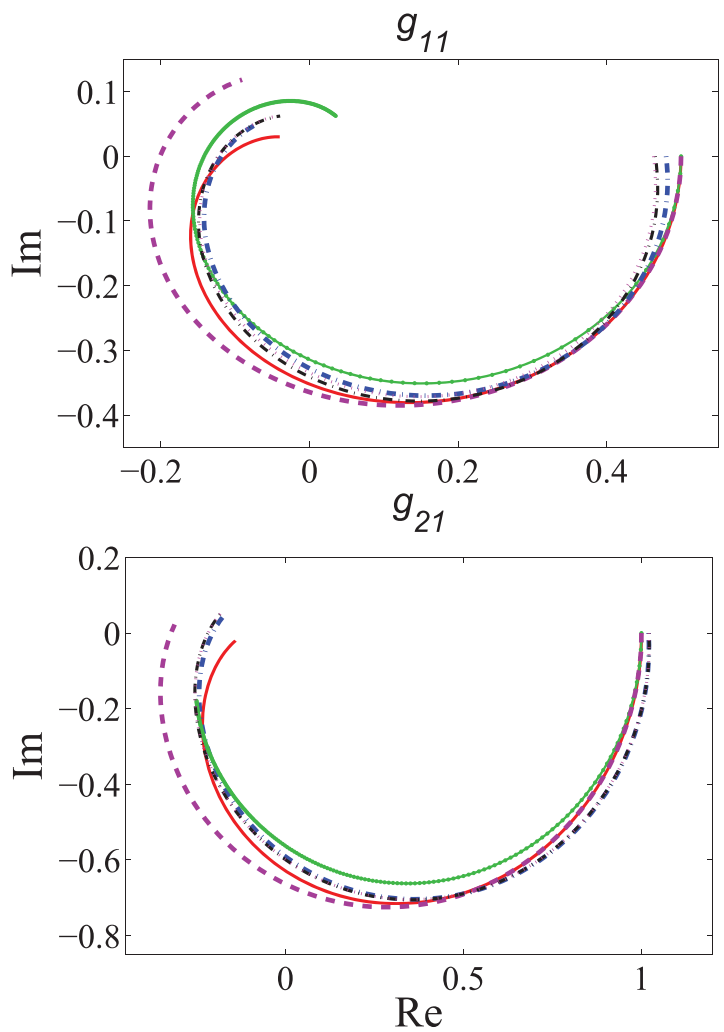
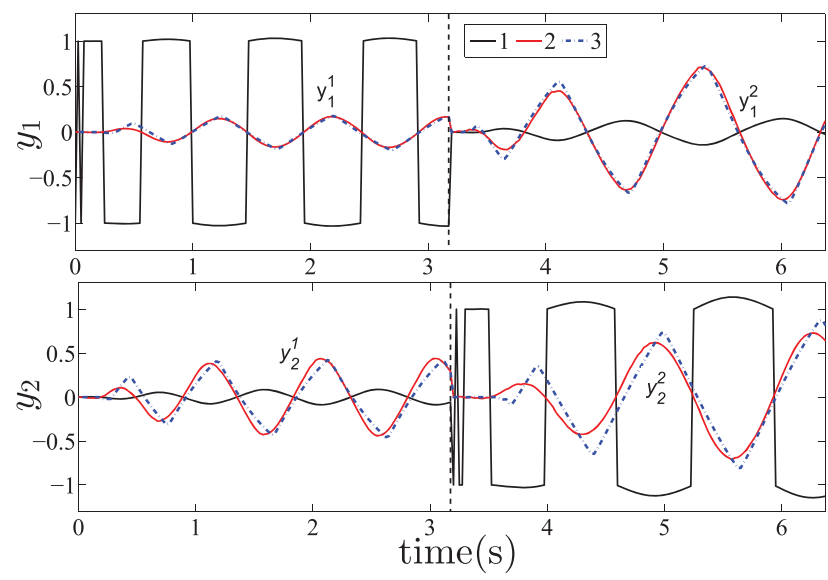

Figure 10. Time responses for $G_{2}$ with $\mathrm{SNR}=40$ : (1) actual and (2) proposed model.

behaviour can be very well estimated only with fractional models.

\subsection{Effect of number of sample points}

The effect of number of samples $M$ is verified for $G_{1}(s)$. Table 2 shows time domain error for various value of $M$. The number of samples is an important factor to
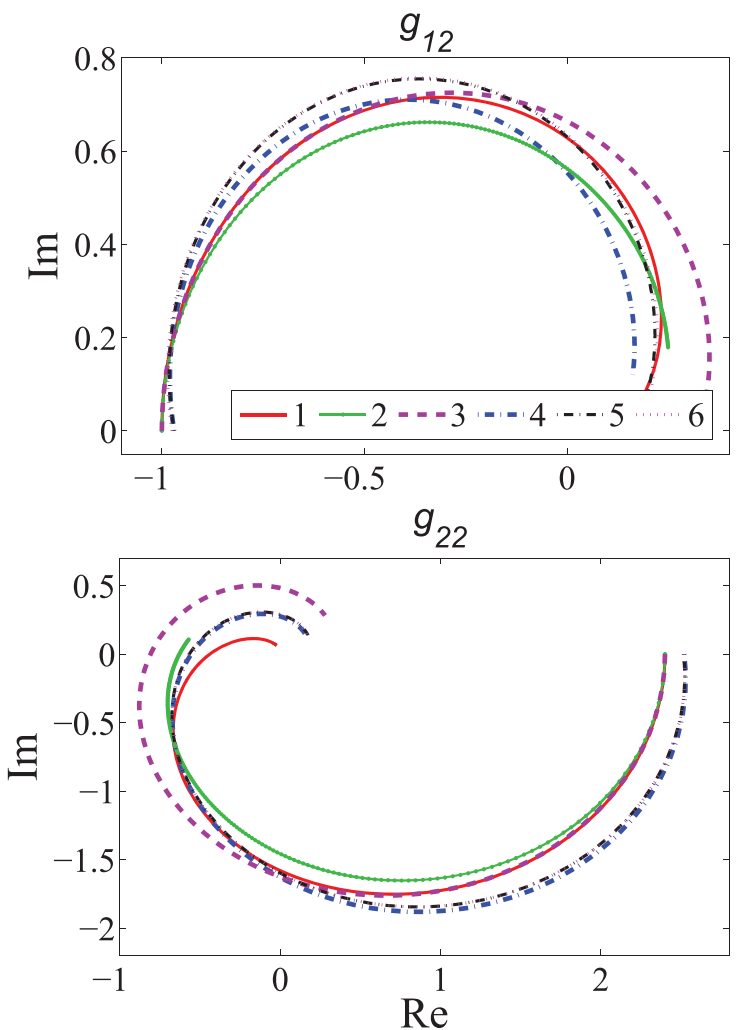

Figure 11. Nyquist plots for $G_{2}$ : (1) actual, (2) SST model, (3) ATT model, (4) proposed model, (5) proposed model (SNR = 20) and (6) proposed model $(\mathrm{SNR}=40)$. 


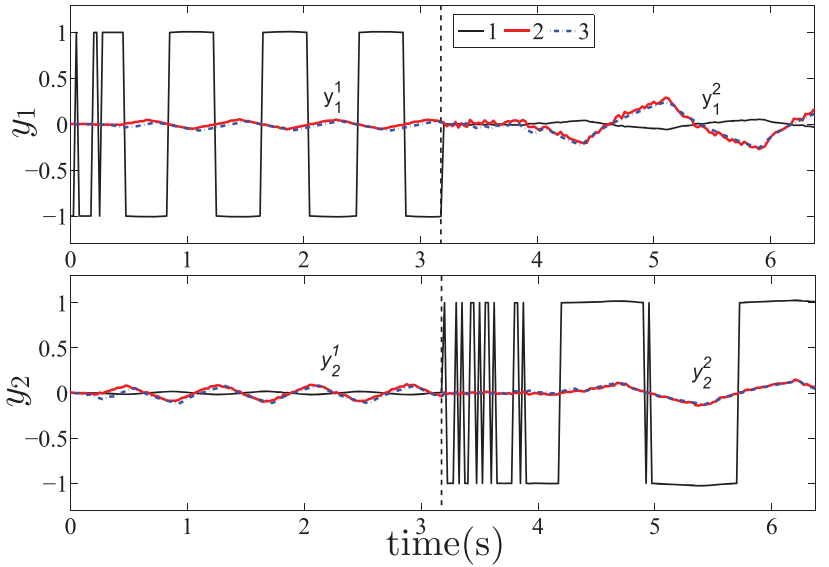

Figure 12. Time responses for $G_{3}$ with $S N R=20 \mathrm{~dB}$ : (1) input, (2) actual and (3) proposed model.

decide the trade-off between the accuracy and speed of the identification. Obviously, a small number of samples accelerates the identification but can result in false estimation. Figure 15 shows normalised mean values of all parameters and their possible range of deviation for different values of $M$. It clearly depicts how the number of samples affects the precision of the identification. Our study recommends for the considered topic the Haar wavelet works well with 256 sample points optimally.
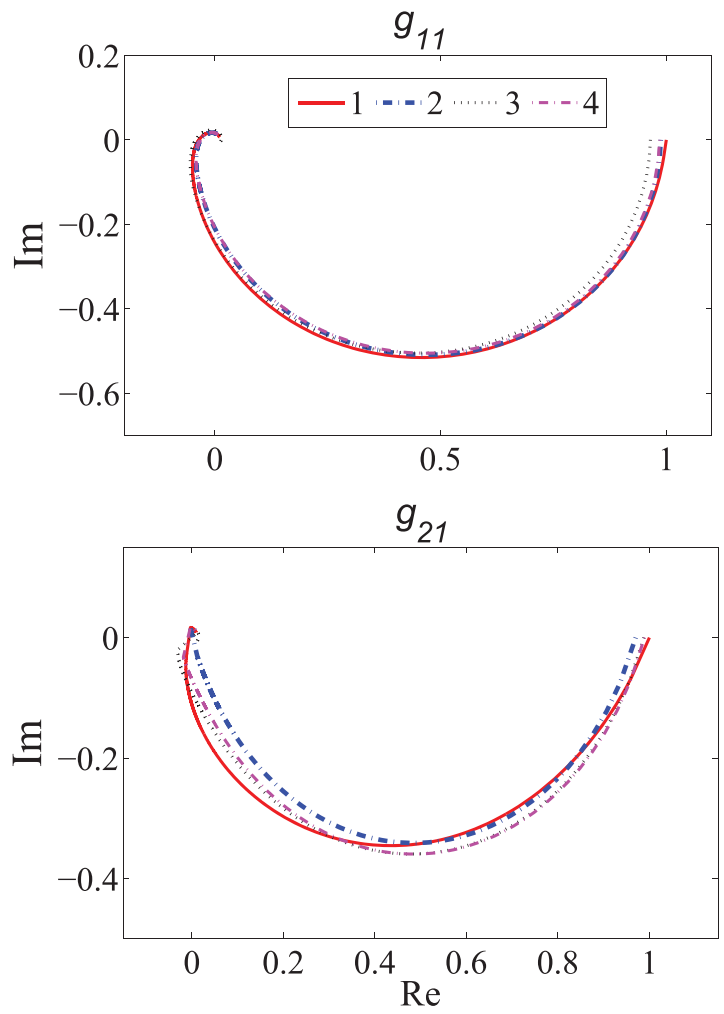

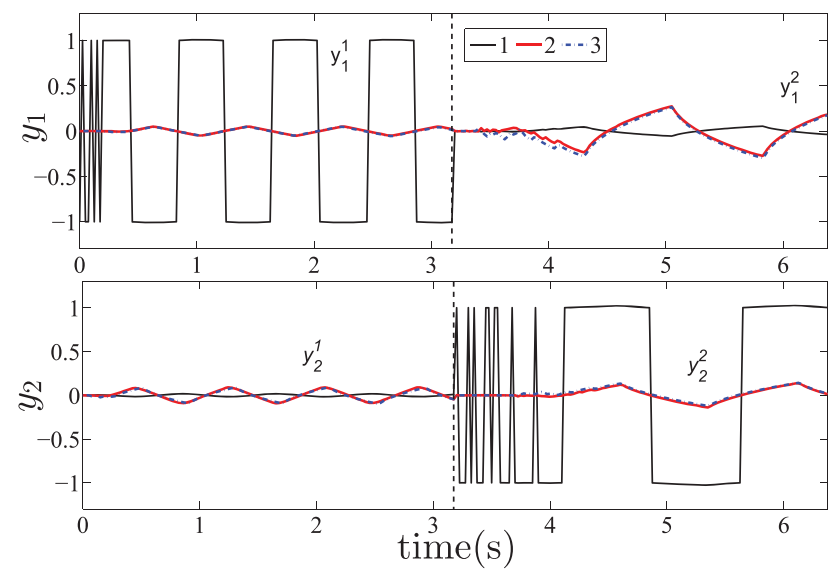

Figure 13. Time responses for $G_{3}$ with $\mathrm{SNR}=40 \mathrm{~dB}$ : (1) input, (2) actual and (3) proposed model.

Table 2. Effect of number of sample points on accuracy.

\begin{tabular}{lc}
\hline Samples & Error $\varepsilon_{t}\left[\begin{array}{ll}y_{1} & y_{2}\end{array}\right] \times 10^{-4}$ \\
\hline$M=512$ & {$\left[\begin{array}{ll}2.12 & 2.25\end{array}\right]$} \\
$M=256$ & {$\left[\begin{array}{ll}3.68 & 4.08\end{array}\right]$} \\
$M=128$ & {$\left[\begin{array}{ll}4.97 & 4.83\end{array}\right]$} \\
$M=64$ & {$\left[\begin{array}{ll}5.73 & 5.87\end{array}\right]$} \\
$M=32$ & {$\left[\begin{array}{ll}33.3 & 40.9\end{array}\right]$} \\
\hline
\end{tabular}

\section{Conclusion}

Provoked by the advantages of Haar operational matrices, this paper has presented a new technique
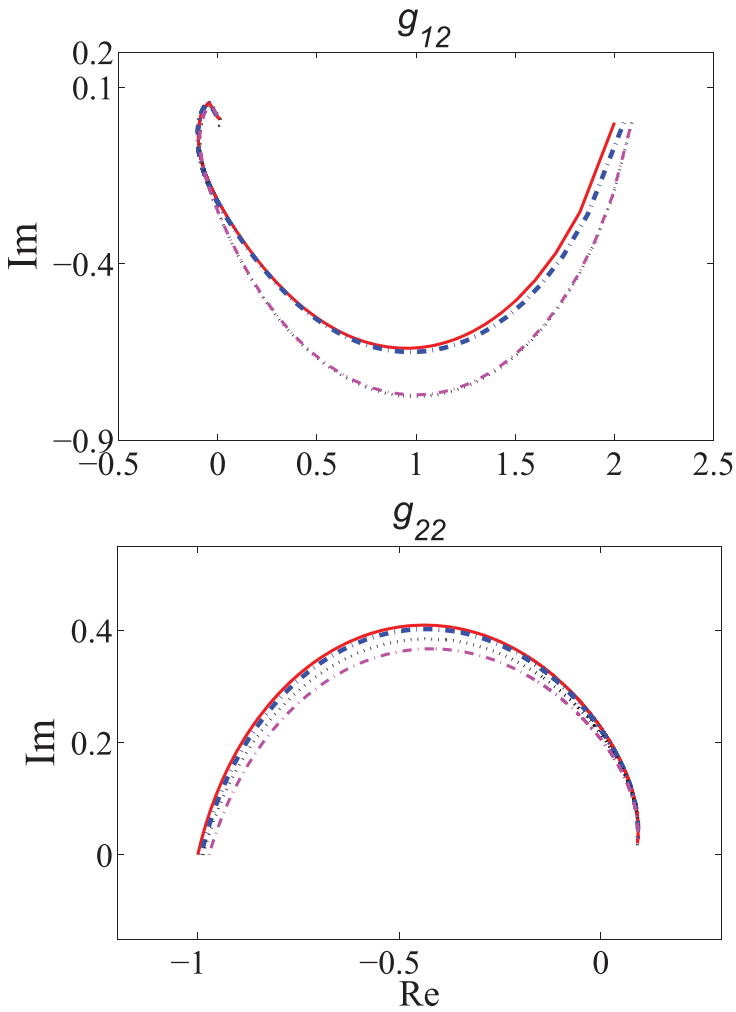

Figure 14. Nyquist plots for $G_{3}$ : (1) actual, (2) proposed model, (3) proposed model (SNR = 20) and (6) proposed model (SNR = 40). 


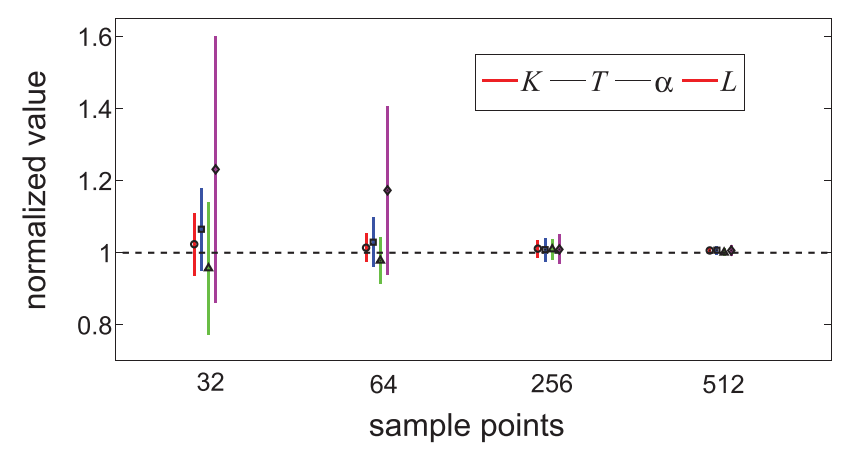

Figure 15. Effect of sample points on parameter identification $\left(g_{11}\right)$. The color of horizontal line before alphabet $T$ should be blue, the color of line before $\alpha$ should be green, and the color of line before $L$ should be purple.

mainly developed for estimating TITO processes. The presented scheme has shown how simply one can achieve a fractional-order model without any fractional derivatives and estimation of the implicit timedelay together with other model parameters. Any closed-loop experiment such as a relay feedback test can be utilised for generating transient data responses. No predecoupling is essential in the identification procedure. Even it does not require to wait for convergence or preprocessing of noisy measured data. Based on simple output and input signals a given unknown TITO process can be identified directly as four FFOTD models. The claim is justified from the verification and analysis of the method on various types of TITO processes. When compared with others, the proposed approach shows overall better accuracy in general even with noisy environment. As it can be seen from the result section, this method identified the higher order process more closely in compared with approach in Nema and Padhy (2015). Moreover, it is evident that the discussed method is based on fractional calculus and can better estimate the fractional-order process dynamics using low-order models compared to other methods (Berner, Soltesz, Hägglund, et al., 2017; S.-Y. Li et al., 2005b; Nema \& Padhy, 2015; Tsay, 2011) which are based on classical calculus. In conclusion, the presented approach is fast, accurate and does not take any priori knowledge of the process.

\section{Disclosure statement}

No potential conflict of interest was reported by the author(s).

\section{Notes on contributors}

Kajal Kothari received the PhD degree in Electrical and Electronics Engineering from the University of the South Pacific, Fiji, and M.Tech. degree in Communication Systems from the Sardar Vallabhbhai National Institute of Technology, India. She is currently working with the School of Engineering and Physics, The University of the South Pacific, Fiji. Her research interests include image processing, system modeling and identification, and fractional systems and control.

Utkal Mehta (SM'15) received the Ph.D. degree from IIT Guwahati, India, in the area of system identification and process control. He is now working in electrical and electronics engineering from The University of the South Pacific, Fiji as an Associate Professor. His current research interests include process identification, applied fractional calculus for modeling, fractional-order filter design on reconfigurable devices like FPAA and various robotics applications for medical and industrial automation.

\section{ORCID}

Kajal Kothari (D) http://orcid.org/0000-0002-5519-9323

Utkal Mehta (D) http://orcid.org/0000-0001-8613-2490

\section{References}

Bajarangbali, \& Majhi, S. (2012). TITO system identification using relay with hysteresis. In 2012 1st international conference on power and energy in NERIST (ICPEN) (pp. 1-5).

Berner, J., Soltesz, K., Åström, K. J., \& Hägglund, T. (2017). Practical evaluation of a novel multivariable relay autotuner with short and efficient excitation. In 2017 IEEE conference on control technology and applications (CCTA) (pp. 1505-1510).

Berner, J., Soltesz, K., Hägglund, T., \& Åström, K. J. (2017). Autotuner identification of TITO systems using a single relay feedback experiment. IFAC PapersOnLine, 50(1), 5332-5337. https://doi.org/10.1016/j.ifacol.2017.08.922

Broman, H., Lindgren, U., Sahlin, H., \& Stoica, P. (1999). A TITO system identification approach. Signal Processing, 73(1), 169-183. https://doi.org/10.1016/S0165-1684(98) 00191-1

Chalupa, P., Přrikryl, J., \& Novák, J. (2015). Modelling of twin rotor MIMO system. Procedia Engineering, 100, 249-258. https://doi.org/10.1016/j.proeng.2015.01.365

Jin, Q., Cheng, Z., Dou, J., Cao, L., \& Wang, K. (2012). A novel closed loop identification method and its application of multivariable system. Journal of Process Control, 22(1), 132-144. https://doi.org/10.1016/j.jprocont.2011.09.008

Jiwari, R. (2012). A Haar wavelet quasilinearization approach for numerical simulation of Burgers' equation. Computer Physics Communications, 183(11), 2413-2423. https://doi. org/10.1016/j.cpc.2012.06.009

Kalpana, D., Thyagarajan, T., \& Gokulraj, N. (2015). Modeling and control of non-square MIMO system using relay 
feedback. ISA Transactions, 59, 408-417. https://doi.org/10.1016/ j.isatra.2015.09.012

Kothari, K., Mehta, U., \& Vanualailai, J. (2018a). A novel approach of fractional-order time delay system modeling based on Haar wavelet. ISA Transactions, 80, 371-380. https://doi.org/10.1016/j.isatra.2018.07.019

Kothari, K., Mehta, U., \& Vanualailai, J. (2018b). Fractionalorder models of time delay systems using Walsh operational matrices. In 2018 15th international conference on control, automation, robotics and vision (ICARCV) (pp. 15551560).

Li, S.-Y., Cai, W.-J., Mei, H., \& Xiong, Q. (2005a). Effective decentralized TITO process identification from closed-loop step responses. Asian Journal of Control, 7(2), 154-162. https://doi.org/10.1111/asjc.2005.7.issue-2

Li, S.-Y., Cai, W.-J., Mei, H., \& Xiong, Q. (2005b). Robust decentralized parameter identification for two-input two-output process from closed-loop step responses. Control Engineering Practice, 13(4), 519-531. https://doi.org/10.1016/j.con engprac.2004.04.017

Li, Z., \& Chen, Y. (2014). Ideal, simplified and inverted decoupling of fractional order TITO processes. IFAC Proceedings, 47(3), 2897-2902. https://doi.org/10.3182/20140824-6-ZA1003.02107

Li, Y., Meng, X., \& Ding, Y.-Q. (2017). Using wavelet multiresolution nature to accelerate the identification of fractional order system. Chinese Physics B, 26(5), 050201. https://doi.org/10.1088/1674-1056/26/5/050201

Li, Y., Meng, X., Zheng, B., \& Ding, Y. (2015). Parameter identification of fractional order linear system based on Haar wavelet operational matrix. ISA Transactions, 59, 79-84. https://doi.org/10.1016/j.isatra.2015.08.011

Mittal, R. C., \& Pandit, S. (2018). Quasilinearized scale-3 Haar wavelets-based algorithm for numerical simulation of fractional dynamical systems. Engineering Computations, 35(5), 1907-1931. https://doi.org/10.1108/EC-09-2017-0347

Monje, C. A., Chen, Y., Vinagre, B. M., Xue, D., \& Feliu-Batlle, V. (2010). Fractional-order systems and controls. SpringerVerlag London.

Nema, S., \& Padhy, P. K. (2015). Identification of two-input twooutput process using state-space analysis. IET Control Theory \& Applications, 9(13), 2029-2038. https://doi.org/10.1049/ iet-cta.2014.1079

Padhy, P. K., \& Majhi, S. (2006). Identification of TITO processes. In 2006 IEEE international conference on industrial technology (pp. 664-669).

Pandit, S., Jiwari, R., Bedi, K., \& Koksal, M. E. (2017). Haar wavelets operational matrix based algorithm for computational modelling of hyperbolic type wave equations. Engineering Computations, 34(8), 2793-2814. https://doi.org/10. 1108/EC-10-2016-0364

Pereira, R. D., Veronesi, M., Visioli, A., Normey-Rico, J. E., \& Torrico, B. C. (2017). Implementation and test of a new autotuning method for PID controllers of TITO processes.
Control Engineering Practice, 58, 171-185. https://doi.org/10. 1016/j.conengprac.2016.10.010

Petráš, I. (2011). Fractional-order nonlinear systems: Modeling, analysis and simulation. Higher Education Press, Beijing and Springer-Verlag Berlin Heidelberg.

Ram, V. D., \& Chidambaram, M. (2014). Closed loop reaction curve method for identification of TITO systems. IFAC Proceedings, 47(1), 989-996. https://doi.org/10.3182/201403133-IN-3024.00135

Ram, V. D., \& Chidambaram, M. (2016). Identification of centralised controlled multivariable systems. Indian Chemical Engineer, 58(3), 240-254. https://doi.org/10.1080/00194506. 2015.1029285

San-Millan, A., Feliu-Talegón, D., Feliu-Batlle, V., \& RivasPerez, R. (2017). On the modelling and control of a laboratory prototype of a hydraulic canal based on a TITO fractional-order model. Entropy 2017, 19(8), 401. https://doi.org/10.3390/e19080401

Semino, D., \& Scali, C. (1998). Improved identification and autotuning of PI controllers for MIMO processes by relay techniques. Journal of Process Control, 8(3), 219-227. https://doi.org/10.1016/S0959-1524(97)00041-3

Tan, K., Lee, T., Huang, S., Chua, K., \& Ferdous, R. (2006). Improved critical point estimation using a preload relay. Journal of Process Control, 16(5), 445-455. https://doi.org/10. 1016/j.jprocont.2005.09.004

Tang, Y., Li, N., Liu, M., Lu, Y., \& Wang, W. (2017). Identification of fractional-order systems with time delays using block pulse functions. Mechanical Systems and Signal Processing, 91, 382-394. https://doi.org/10.1016/j.ymssp.2017. 01.008

Tastemirov, A., Lecchini-Visintini, A., \& Morales-Viviescas, R. M. (2017). Complete dynamic model of the twin rotor MIMO system (TRMS) with experimental validation. Control Engineering Practice, 66, 89-98. https://doi.org/10.1016/ j.conengprac.2017.06.009

Tsay, T.-S. (2011). On-line tuning of PI/Lead compensators for decoupling multivariable processes with specifications on phase and bandwidth. Journal of the Franklin Institute, 348(9), 2586-2606. https://doi.org/10.1016/j.jfranklin.2011. 07.018

Viswanathan, P. K., Toh, W. K., \& Rangaiah, G. P. (2001). Closed-loop identification of TITO processes using timedomain curve fitting and genetic algorithms. Industrial \& Engineering Chemistry Research, 40(13), 2818-2826. https:// doi.org/10.1021/ie000135d

Xue, D., \& Li, T. (2018). An approach to design controllers for MIMO fractional-order plants based on parameter optimization algorithm. ISA Transactions, 82, 145-152. https://doi.org/10.1016/j.isatra.2017.04.022

Zheng, J., Guo, G., \& Wang, Y. (2005). Identification and decentralized control of a dual-actuator hard disk drive system. IEEE Transactions on Magnetics, 41(9), 2515-2521. https://doi.org/10.1109/TMAG.2005.855442 